\title{
Prognostic significance of tumor-infiltrating lymphocytes in ductal carcinoma in situ of the breast
}

\author{
Michael S. Toss $\mathbb{1}^{1,2} \cdot$ I. Miligy ${ }^{1,3} \cdot$ Abdubaqi Al-Kawaz $^{1} \cdot$ Mansour Alsleem ${ }^{1} \cdot$ Hazem Khout $^{1,4} \cdot$ Padmashree C. Rida ${ }^{5} \cdot$ \\ Ritu Aneja ${ }^{5}$. Andrew R. Green ${ }^{1} \cdot$ Ian O. Ellis ${ }^{1,4} \cdot$ Emad A. Rakha ${ }^{1,3,4}$
}

Received: 13 October 2017 / Revised: 31 January 2018 / Accepted: 31 January 2018 / Published online: 20 March 2018

(c) United States \& Canadian Academy of Pathology 2018

\begin{abstract}
Tumor-infiltrating lymphocytes (TILs) provide prognostic value in invasive breast cancer and guidelines for their assessment have been published. This study aims to evaluate: (a) methods of TILs assessment, and (b) their prognostic significance in breast ductal carcinoma in situ (DCIS). Hematoxylin and eosin sections from two clinically annotated DCIS cohorts; a training set $(n=150$ pure DCIS) and a validation set $(n=666$ comprising 534 pure DCIS and 132 cases wherein DCIS and invasive breast carcinoma were co-existent) were assessed. Seven different scoring methods were applied to the training set to identify the most optimal reproducible method associated with strongest prognostic value. Among different methods, TILs touching ducts' basement membrane or away from it by one lymphocyte cell thickness provided the strongest significant association with outcome and highest concordance rate [inter-cluster correlation coefficient $=0.95$ ]. Assessment of periductal TILs at increasing distances from DCIS $(0.2,0.5$, and $1 \mathrm{~mm})$ as well as percent of stromal TILs were practically challenging and showed lower concordance rates than touching TILs. TILs hotspots and lymphoid follicles did not show prognostic significance. Within the pure DCIS validation set, dense TILs were associated with younger age, symptomatic presentation, larger size, higher nuclear grade, comedo necrosis and estrogen receptor negativity as well as shorter recurrence-free interval $(p=0.002)$. In multivariate survival analysis, dense TILs were independent predictor of shorter recurrence-free interval $(p=0.002)$ in patients treated with breast conservation. DCIS associated with invasive carcinoma showed denser TILs than pure DCIS $\left(p=9.0 \times 10^{-13}\right)$. Dense TILs is an independent prognostic variable in DCIS. Touching TILs provides a reproducible method for their assessment that can potentially be used to guide management
\end{abstract}

Electronic supplementary material The online version of this article (https://doi.org/10.1038/s41379-018-0040-8) contains supplementary material, which is available to authorized users.

Emad A. Rakha

emad.rakha@nottingham.ac.uk

Emad.rakha@nuh.nhs.uk

1 Academic Pathology, Division of Cancer and Stem Cells, School of Medicine, The University of Nottingham, Nottingham, UK

2 Histopathology department, South Egypt Cancer Institute, Asyut University, Asyut, Egypt

3 Histopathology department, Faculty of Medicine, Menoufia University, Shebin El-Kom, Egypt

4 Breast Institute, Nottingham University Hospitals NHS Trust, Nottingham City Hospital, Hucknall Road, Nottingham NG5 1PB, UK

5 Department of Biology, Georgia State University, Atlanta, GA USA

\section{Introduction}

The incidence of ductal carcinoma in situ (DCIS) of the breast has dramatically increased after introduction of mammographic screening programs $[1,2]$. This, together with increasing use of breast-conserving surgery for management of breast cancer, has heightened the need for robust and reliable predictors of disease recurrence and progression, for risk-stratification of patients. Comparison of DCIS recurrence rates and progression (to invasive disease) with the rates of mastectomies, surgical re-excisions or intense course of radiotherapy suggests overtreatment, stemming from a lack of accurate risk-stratification [3]. Current application of molecular genetic signatures for DCIS categorization to provide suitable individualized management remains challenging [4-9].

Tumor-infiltrating lymphocytes (TILs) are indicators of the adaptive immune response against tumors and play a cornerstone role in cancer immunotherapy [10, 11]. In 
previous studies on invasive breast cancer, dense TILs were shown to augment effect of chemotherapy providing better prognosis mainly in triple-negative breast tumors [12, 13]. Following demonstration of their prognostic significance in invasive breast carcinoma and their potential clinical application, guideline recommendations for TILs evaluation have been published [14, 15]. However, the recommended method of TILs assessment in invasive breast carcinoma may not be applicable in DCIS as not all stromal TILs are directly in contact with malignant ducts and identification of stromal area surrounding DCIS can be confusing and illdefined. Despite the reported role of TILs in DCIS [5, 16], studies utilizing the invasive carcinoma guidelines for TILs assessment in the context of DCIS did not find any association with outcome $[15,17]$. This is likely related to the difference in nature and distribution of TILs within DCIS compared to invasive breast carcinoma and the sub-optimal method of their assessment. This study aims to identify the optimal method of TILs evaluation in DCIS in terms of reliability, reproducibility and prognostic significance with recurrence through utilization of a large well-annotated DCIS cohort with long-term follow-up.

\section{Materials and methods}

\section{Study cohort}

This retrospective study included 982 cases diagnosed from 1990 to 2012 at Nottingham City Hospital, Nottingham, United Kingdom. Following histological review of tumor slides of all cases and retrieval of tissue blocks, representative hematoxylin and eosin-stained sections from each case were prepared from 816 cases (684 pure DCIS and 132 DCIS mixed with invasive carcinoma). Representative sections were defined as those having the largest tumor burden. Clinicopathological data included patients' age, type of presentation (whether symptomatic or screendetected), DCIS tumor size, nuclear grade, presence of comedo necrosis, associated Paget's disease, detailed information about management, and outcome including surgery type, and number of operations, local radiotherapy treatment, occurrence, and nature of local recurrence and recurrence-free interval were collected from local data recording systems. Recurrence-free interval is defined as time between first DCIS surgery and occurrence of ipsilateral tumor recurrence (either as DCIS or invasive carcinoma) in months. Cases with contralateral breast event or invasive breast carcinoma developed as a new primary tumor in a different quadrant were censored at the time of recurrences. For molecular characterization of DCIS, estrogen receptor immunohistochemical staining was performed in cases with available paraffin blocks. Four micrometer sections were stained on the Ventana BenchMark $^{\circledR}$ ULTRA system (Tucson, Arizona, USA) using Ventana estrogen receptor (SP1) Rabbit Monoclonal Primary Antibody as per recommended protocol.

The cohort was split into two-groups: a training set and a validation set (clinicopathological parameters of both sets are shown in Supplementary Table 1):

\section{Training set}

The training set included 150 pure DCIS from patients older than 50 years of age with mixture of DCIS grades that was completely excised with free surgical margins $(10 \mathrm{~mm}$ or more) to avoid the confounding effect of age or margin of excision on the outcome analyses. Within a median followup period of 161 months; 41 cases (27\%) developed ipsilateral recurrence.

\section{Validation set}

The validation set $(n=666)$ was further split into two subgroups: (1) Pure DCIS $(n=534)$, which showed 63 ipsilateral local recurrence events $(11.8 \%)$ within a median follow-up period of 109 months; (2) DCIS with co-existent invasive breast carcinoma $(n=132)$ to compare the pattern of TILs density between pure DCIS cases and cases wherein DCIS is associated with invasion. Supplementary Fig. 1 shows the algorithm of the study cohorts.

\section{Scoring of TILs}

Freshly stained hematoxylin and eosin (4-5 $\mu \mathrm{m}$ thick) fullface sections were scanned using a high-resolution slide scanner (PANNORAMIC 250 FLASH III, 3D-HISTECH), followed by viewing the slides using "Pannoramic Viewer Software program, version 1.15.4." In this study, TILs were counted manually (eyeballing) using digital images. The International Working Group Recommendations for TILs assessment were modified and applied to our case series [15]. All recognizable mononuclear inflammatory cells including lymphocytes and plasma cells were counted (polymorphonuclear cells were excluded). TILs were assessed around all DCIS duct profiles up to 20 ducts (the average number of ducts within the training set). For cases with more than 20 malignant ducts, we divided the section field into four identical quadrants using the Pannoramic Viewer software, and scored TILs around five ducts in each quadrant in order to keep the scoring more representative especially in cases with heterogeneously distributed TILs. During scoring, very large (i.e., mass forming papillary carcinoma, branching or confluent DCIS ducts) or very small (terminal duct-lobular units involved by DCIS) ducts were not considered in TILs scoring to avoid skewing of the 
Table 1 Methods and parameters of tumor-infiltrating lymphocytes (TILs) assessment in DCIS

Methods for evaluation of TILs in DCIS

(1) TILs were assessed in hematoxylin and eosin-stained sections. Only full-face sections from surgically excised specimen were assessed. Lesions diagnosed on core biopsy were not included (2) One representative section $(4 \mu \mathrm{m})$, per patient, which has the largest tumor burden, was selected for TILs assessment

(3) All mononuclear inflammatory cells apart from polymorphonuclear leukocytes were counted

(4) TILs within the boundaries of the DCIS were assessed. TILs beyond the tumor limits, surrounding normal ducts/lobules, adjacent fatty tissue, lobular carcinoma in situ, regressive hyalinosis, crushed artefacts or sites of previous biopsy were excluded

(5) TILs within tumor cells (intra-tumor TILs) were not assessed (6) TILs were assessed around all malignant ducts up to 20 ducts. For lesions with more than 20 malignant ducts, we assessed TILs surrounding 20 ducts (five ducts from each quadrant of the lesion) (7) TILs were assessed around average sized ducts only (case specific). TILs around very large DCIS ducts such as mass forming papillary carcinoma, branching or confluent DCIS ducts or very small ducts such as terminal duct-lobular system involved by DCIS were excluded

(8) Any TILs infiltrating the ducts' circumference were considered. Overlapping TILs between adjacent ducts were counted once

Parameters used for TILs assessment ${ }^{\mathrm{a}}$

(A) Estimation of stromal TILs (as previously published):

- The stromal area was defined as the area surrounding the DCIS duct within two high-power microscopic fields and used for evaluation of stromal TILs percentage $[15,17,18]$. In cases with numerous involved ducts, an evaluation of the area surrounding the whole lesion was performed, and percentage of stromal TILs in the total stromal area of all DCIS involved ducts was determined [5, 15, 19]

(B) Estimation of periductal TILs (based on counting TILs around all DCIS duct profiles up to 20 ducts)

(1) Evaluation of the mean number of TILs touching DCIS involved ducts (defined as TILs touching or within one lymphocyte cell thickness from ducts' basement membrane)

(2) Evaluation of the mean number of TILs within $0.2 \mathrm{~mm}$ distance from the ducts

(3) Evaluation of mean number of TILs within $0.5 \mathrm{~mm}$ distance from the ducts

(4) Evaluation of mean number of TILs with $1.0 \mathrm{~mm}$ distance from the ducts

(5) Evaluation of the TILs hotspot defined by largest number of lymphoid aggregates directly surrounding or located between DCIS ducts within the boundaries of the lesion

(6) Evaluation of lymphoid follicles formations with reactive germinal centers in the stroma directly surrounding or located between DCIS ducts within the boundaries of the lesion

${ }^{a}$ All parameters were assessed in the training set while touching and stromal TILs assessment were conducted to validation set

scores by the effect of extremes in duct sizes. Pure encapsulated papillary carcinomas, not associated with adjacent DCIS, were excluded from the scoring. Any type of circumferential TILs-infiltration was considered, including minimal, partial, subtotal, and total circumferential TILsinfiltration around ducts. TILs beyond the lesion limits, surrounding the adjacent or intermingled fat, normal ducts, associated lobular carcinoma in situ, regressive hyalinosis or at crushing artefacts were excluded from the scoring.

In the training set, TILs were assessed using seven different scoring methods (Table 1). These included: (1) Percentage of stromal TILs (assessed in a manner similar to the modified method for evaluation of TILs in invasive breast carcinoma. The stromal area was defined as the area surrounding the DCIS duct within two high-power microscopic fields and used for evaluation of stromal TILs percentage $[15,17,18]$. In cases with numerous involved ducts, an evaluation of the area surrounding the whole lesion was performed, and percentage of stromal TILs in the total stromal area of all DCIS involved ducts was determined [5, 15, 19], (2) The mean number of touching TILs defined by TILs touching or within one lymphocyte cell thickness from the malignant ducts' basement membrane; (3) Mean number of TILs within $0.2 \mathrm{~mm}$ distance from ducts' basement membranes; (4) Mean number of TILs located within $0.5 \mathrm{~mm}$ distance from ducts' basement membranes; (5) Mean number of TILs located within $1 \mathrm{~mm}$ from ducts' basement membranes; (6) TILs hotspots defined as the largest number of lymphoid cells aggregates directly surrounding or located between DCIS ducts within the boundaries of the lesion; and (7) Assessment of lymphoid follicles with reactive germinal centers. Counting of TILs at different topographic areas was carried out manually with aid of the Panoramic Viewer Software program scale. Overlapping TILs between adjacent ducts in each topographic area were counted once. The detailed methods followed to assess TILs are summarized in Table 1 and illustrated in Fig. 1.

To check the reliability and reproducibility of the evaluation methods, training set was scored using all the previous parameters by three observers (MST, IMM, and AK). Two observers discussed the detailed methodology before starting their scoring, while the third observer scored the cases based on a written protocol without any verbal discussion. The optimal method for TILs assessment was determined based on the association with outcome and reproducibility in terms of inter-observer concordance as well as practicality, which was then applied to the validation set. Validation set, including the pure DCIS and mixed cohorts, was scored for touching TILs by two observers (MST and AK) to confirm reproducibility and prognostic significance. In mixed cases, touching TILs were scored around the DCIS component only while TILs adjacent to invasive tumor were not considered in the scoring. TILs scoring carried out by the first observer (MST) were considered in the final statistical analysis (the main researcher for this study). Other observers' scores were used to check the reproducibility and concordance rate. 
Fig. 1 Parameters of TILs assessment. a Touching lymphocytes (x40) defined by lymphocytes that touch the basement membrane (BM) or are located within one lymphocyte cell thickness distance from basement membrane (yellow arrows); inset closer view for touching TILs, $\mathbf{b}$ TILs assessment within $0.2,0.5$, and $1.0 \mathrm{~mm}$ distance from the involved ducts, and $\mathbf{c}$ evaluation of hotspots (largest number of lymphoid cells aggregates within the lesion as shown in area surrounded by black dashed circle)
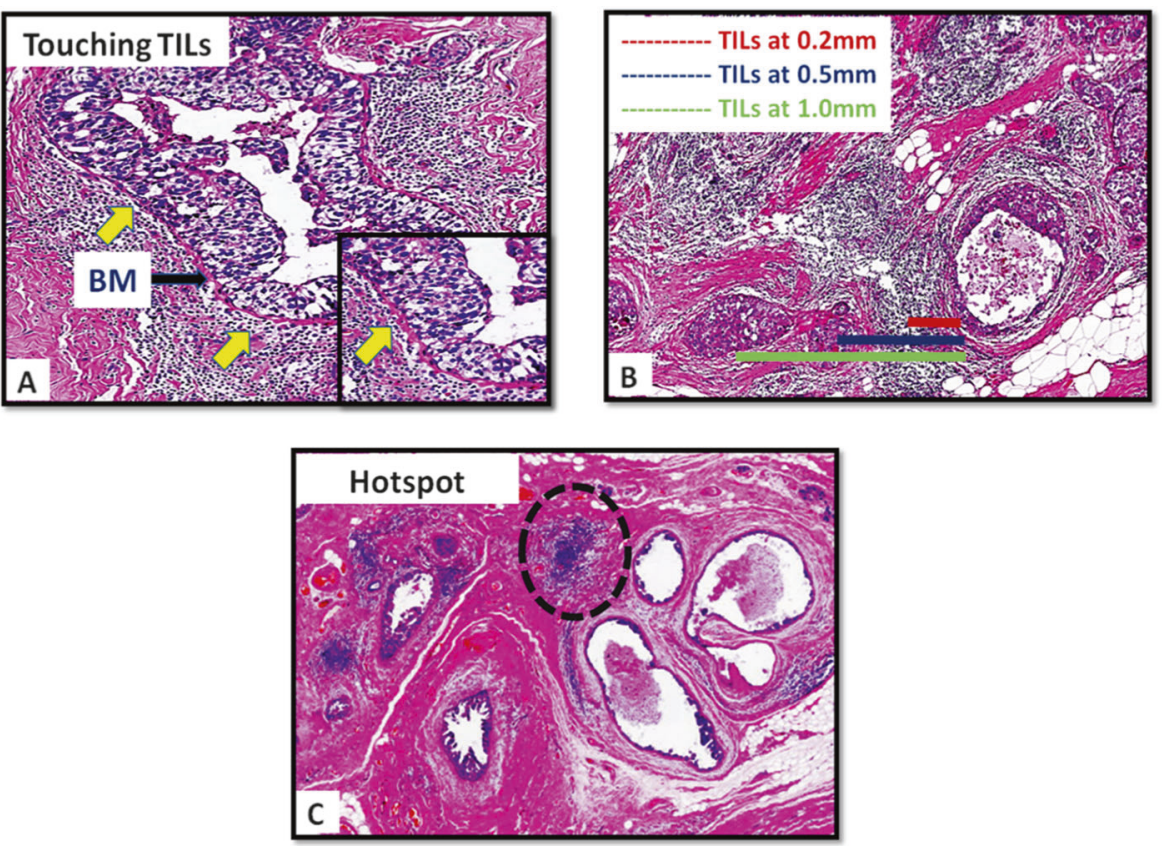

As assessment of stromal TILs was the method used to evaluate TILs in DCIS in previous studies [15, 17, 18], stromal TILs was also evaluated in the pure DCIS validation set using the same criteria applied in training set.

This study is ethically approved by the North WestGreater Manchester Central Research Ethics Committee (15/NW/0685).

\section{Statistical analysis}

The optimal cutoff point for TILs density against recurrence-free interval was defined using X-tile bioinformatics software (Yale University, version 3.6.1). TILs were classified into sparse infiltrates and dense infiltrates depending on these cutoff points (Supplementary Table 2). For consistency, the same cutoff point used in dichotomization touching TILs in the training set into sparse and dense groups was applied in the validation set. Furthermore, to mimic the three-tier prognostic classification system of TILs in melanoma [20] and based on the outcome analysis, TILs were further defined into three-groups; absent/very scanty (mean number of touching TILs/DCIS duct $\leq 5$ cells), sparse (6-20 cells/DCIS duct) and dense ( $>20$ cells/DCIS duct) TILs. This was based on counting TILs around DCIS ducts, in up to 20 ducts per case, and then the total number of TILs was divided by the number of DCIS ducts resulting in the mean TILs number. IBM-SPSS statistical software 22.0 (SPSS, Chicago, IL, USA) was used to analyze our findings. Inter-observer degree of agreement was assessed through inter-class correlation coefficient for continuous data and Kappa test for categorical groups. Linear correlation between TILs densities within different topographic areas and touching TILs was analyzed using Spearman's test. Kaplan-Meier curves and log-rank test were used for univariate survival analyses while Cox regression model was used for the multivariate analysis for patients treated with breast-conserving surgery. Two tailed $p$-value $<0.05$ was considered as statistically significant.

\section{Results}

This study included two sets of cases: a training set comprising 150 pure DCIS cases scored by three observers using seven different scoring methods and a validation set ( $n=666$ cases comprising 534 pure DCIS and 132 DCIS cases with co-existing invasive carcinoma), which was scored for touching and stromal TILs by two observers.

\section{Training set}

The concordance rate of TILs assessment using different methods between the three observers is summarized in Supplementary Table 3. The highest degree of interobserver agreement was observed in touching TILs (Intercluster correlation coefficient $=0.96$ ) whereas the least concordance rate was found with the percentage of stromal TILs evaluation (Inter-cluster correlation coefficient $=$ 0.79 ), which is the recommended method in invasive breast carcinoma.

Mean TILs counts within different topographic areas around DCIS are summarized in Supplementary Table 4. The mean count of TILs increased from 37 cells/DCIS duct at touching area to 482 cells/DCIS duct within $1 \mathrm{~mm}$ 
Table 2 Frequency of TILs density in different topographic areas and their association with outcome in terms of recurrence-free interval in the training set

\begin{tabular}{|c|c|c|c|c|}
\hline Parameter & $\begin{array}{l}\text { TILs density (mean number } \\
\text { of TILs/DCIS duct as cutoff) }\end{array}$ & $\begin{array}{l}\text { Number of } \\
\text { cases }(\%)\end{array}$ & Recurrence $(\%)$ & $\begin{array}{l}p \text {-value }(\log \text { - } \\
\text { rank test) }\end{array}$ \\
\hline \multirow{2}{*}{$\begin{array}{l}\text { Touching TILs (two- } \\
\text { tier system) }\end{array}$} & Sparse $(\leq 20)$ & $70(47)$ & $6(15)$ & \multirow[t]{2}{*}{$4.7 \times 10^{-6}$} \\
\hline & Dense $(>20)$ & $80(53)$ & $35(85)$ & \\
\hline \multirow{3}{*}{$\begin{array}{l}\text { Touching TILs (three- } \\
\text { tier system) }\end{array}$} & Absent/very scanty $(\leq 5)$ & $7(5)$ & $0(0)$ & \multirow[t]{3}{*}{$2.0 \times 10^{-5}$} \\
\hline & Sparse (6-20) & $63(42)$ & $6(15)$ & \\
\hline & Dense $(>20)$ & $80(53)$ & $35(85)$ & \\
\hline \multirow{2}{*}{$\begin{array}{l}\text { TILs at } 0.2 \mathrm{~mm} \\
\text { distance }\end{array}$} & Sparse $(\leq 60)$ & $64(43)$ & $8(20)$ & \multirow[t]{2}{*}{0.001} \\
\hline & Dense $(>60)$ & $86(57)$ & $33(80)$ & \\
\hline \multirow{2}{*}{$\begin{array}{l}\text { TILs at } 0.5 \mathrm{~mm} \\
\text { distance }\end{array}$} & Sparse $(\leq 100)$ & $61(41)$ & $8(20)$ & \multirow[t]{2}{*}{0.002} \\
\hline & Dense $(>100)$ & 89 (59) & $33(80)$ & \\
\hline \multirow{2}{*}{$\begin{array}{l}\text { TILs at } 1.0 \mathrm{~mm} \\
\text { distance }\end{array}$} & Sparse $(\leq 120)$ & $53(35)$ & $6(15)$ & \multirow[t]{2}{*}{0.001} \\
\hline & Dense $(>120)$ & $97(65)$ & $35(85)$ & \\
\hline \multirow[t]{2}{*}{ Hotspot } & Sparse $(\leq 1200)$ & $103(69)$ & $33(80)$ & \multirow[t]{2}{*}{0.089} \\
\hline & Dense $(>1200)$ & $47(31)$ & $8(20)$ & \\
\hline \multirow[t]{2}{*}{ Lymphoid follicles } & No & $119(79)$ & $36(88)$ & \multirow[t]{2}{*}{0.150} \\
\hline & Yes & $31(21)$ & $5(12)$ & \\
\hline \multirow{2}{*}{$\begin{array}{l}\text { Percentage of stromal } \\
\text { TILs }\end{array}$} & Sparse $(\leq 5 \%)$ & $77(51)$ & $15(37)$ & \multirow[t]{2}{*}{0.020} \\
\hline & Dense $(>5 \%)$ & $73(49)$ & $26(63)$ & \\
\hline
\end{tabular}

Significant $p$ values are in bold

distance. Largest hotspot density ranged from 20 to 6000 cells while percentage of stromal TILs ranged from 1-65\%. Mean TILs density increased by fourfold between touching and within $0.2 \mathrm{~mm}$ distance, while it increased only by twofold and 1.5 -fold from 0.2 to $0.5 \mathrm{~mm}$ and from 0.5 to 1 $\mathrm{mm}$ distances, respectively. No cases with absent TILs were observed. Touching TILs showed positive linear correlation with TILs within the other topographic areas (Supplementary Table 5). The highest correlation was observed between touching TILs and TILs counted at $0.2 \mathrm{~mm}$ distance (Spearman's correlation $=$ $\left.0.854, p=1 \times 10^{-13}\right)$. Counting TILs in farer areas away from the ducts showed less correlation with touching TILs.

Percentage of the cases with dense TILs were 53\%, 57\%, $59 \%$, and $65 \%$ for touching TILs and within $0.2,0.5$, and 1 $\mathrm{mm}$ from DCIS ducts, respectively. Dense hotspots were observed in $31 \%$ of cases while dense stromal TILs were observed in $49 \%$ of cases. Lymphoid follicles were observed in 31 cases only (21\%). The percentages of cases with dense and sparse TILs in context of each topographic area as well as the percentage of cases that developed local recurrence within each category and their association with recurrence-free interval are summarized in Table 2. Examples of dense and sparse TILs are shown in Fig. 2.

More than $60 \%$ of DCIS cases that developed local recurrence showed dense TILs irrespective of the topographic area used for assessment (touching, $0.2 \mathrm{~mm}$ distance, $0.5 \mathrm{~mm}, 1 \mathrm{~mm}$ and $\%$ stromal TILs). Only five cases (12\%) from those developed local recurrence showed lymphoid follicle formations. Dense TILs infiltrate at different compartments showed statistically significant association with shorter recurrence-free interval $\left(p=4.7 \times 10^{-6}\right.$, $p=0.001, p=0.002$, and $p=0.001$ for touching TILs and TILs within $0.2,0.5$, and $1 \mathrm{~mm}$ distances, respectively). Stromal TILs density was also associated with shorter recurrence-free interval but with less statistical significance $(p=0.02)$ than dense TILs defined in context of circumferential distances from the malignant ducts. Neither hotspots nor lymphoid follicles formation showed significant association with recurrence $(p=0.09$ and $p=0.15$, respectively). Results of univariate survival analysis are detailed in Table 2. Supplementary Fig. 2 shows Kaplan-Meier survival curves for TILs density in different compartments and recurrence-free interval.Please check if \&\#x00027;(touching, 0.2\&\#x02009;mm distance, $0.5 \& \# \mathrm{x} 02009 ; \mathrm{mm}, 1 \& \# \mathrm{x} 02009 ; \mathrm{mm}$ and \% stromal TILs). $\& \# \mathrm{x} 00027$; is correct here.Yes, they are correct and consistent with the supplementary figure

\section{Validation set}

The concordance rate between the two observers evaluated touching TILs in the validation set was comparable to the training set (Inter-cluster correlation coefficient $=0.95$ ). 
Fig. 2 Touching TILs density around DCIS. a Dense infiltrate where mean number of touching TILs is more than 20 cells/DCIS duct, b High-power view for dense touching TILs, and c Sparse infiltrate where the mean number of touching TILs within the lesion is 20 cells or less/ DCIS duct
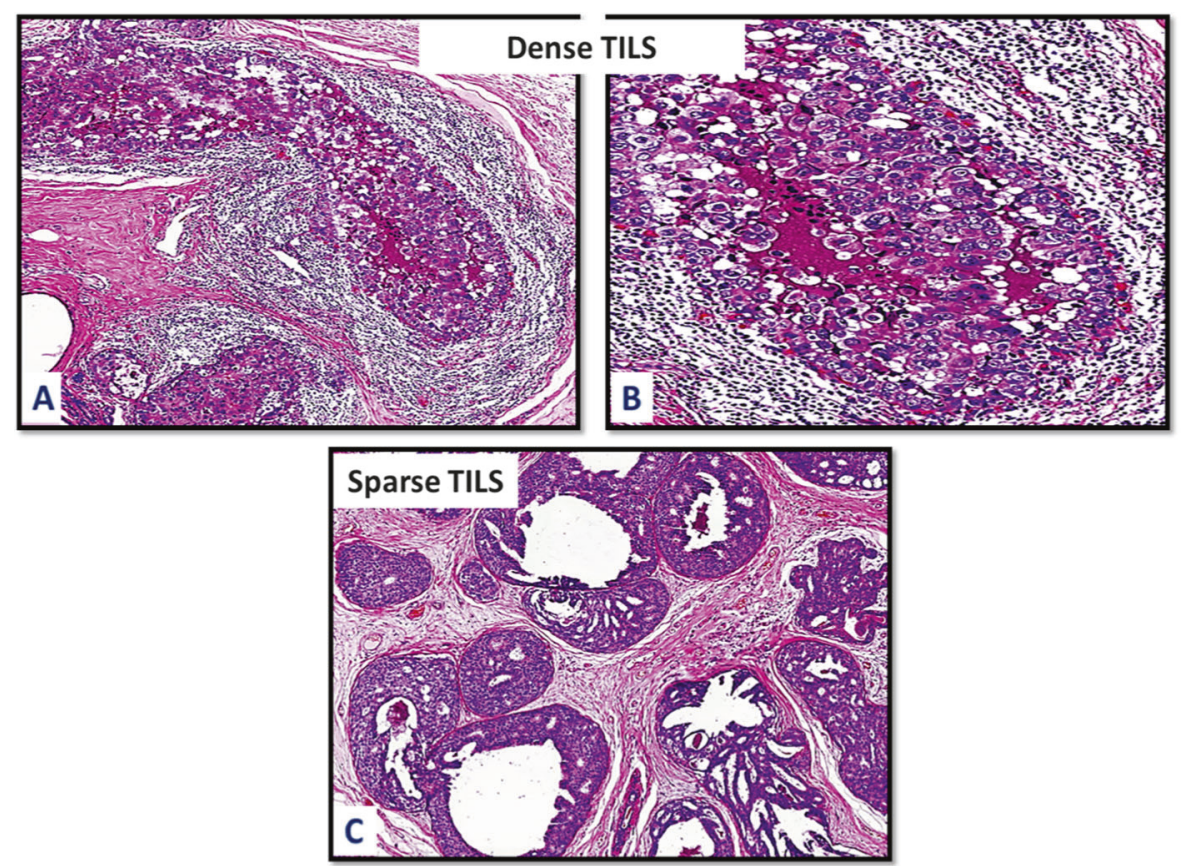

Dichotomization of touching TILs using 20 cells/DCIS duct as a cutoff point, showed that 239 cases $(45 \%)$ had dense TILs infiltrate while $55 \%$ of cases showed sparse infiltrate.

\section{Association of TILs density with other clinicopathological parameters}

Dense touching TILs were associated with parameters characteristic of aggressive tumor behavior including younger age, symptomatic presentation, and larger tumor size. Only eight cases (14\%) of low nuclear grade lesions showed dense TILs in comparison with $33 \%$ and 54\% of intermediate and high nuclear grade lesions, respectively $\left(p=1.1 \times 10^{-9}\right)$. Half of the cases $(50 \%)$ associated with comedo necrosis harbored dense TILs while this was observed only in $32 \%$ of cases without comedo necrosis $(p=0.00009)$. Approximately two thirds $(69 \%)$ of DCIS cases associated with Paget's disease showed dense TILs infiltrate compared with $46 \%$ of patients without Paget's disease $(p=0.025)$. Seventy-two percent of estrogen receptor-negative DCIS showed dense TILs infiltrate while only $36 \%$ of estrogen receptor-positive cases showed high TILs density $\left(p=1.04 \times 10^{-10}\right)$.

Moreover, dense TILs were observed in $80 \%$ of DCIS mixed with invasion compared with $54 \%$ of pure gradematched DCIS $\left(\chi^{2}=51.29, p=8.96 \times 10^{-13}\right)$. This association was observed also when touching TILs was assessed as a continuous variable $\left(p=3.14 \times 10^{-12}\right)$ (Supplementary Fig. 3). The association between TILs density and various clinicopathological parameters are summarized in Table 3.

\section{Outcome analysis}

Ipsilateral local recurrence rate in the pure DCIS validation cohort was $11.8 \%(n=63)$. Recurrence occurred in 39/239 cases with dense TILs (62\% from total recurrences), in comparison with $24 / 295$ cases with sparse TILs (38\% from total recurrences). Within patients treated with breastconserving surgery, local recurrence was reported in 24/80 cases with dense TILs (56\%) compared to 19/139 cases with sparse infiltrate (44\%). When assessed based on estrogen receptor status, there were 22 recurrent events among estrogen receptor-positive DCIS with dense TILs ( $n$ $=114$ ) compared with 40 events among cases with sparse TILs $(n=198)$. Among estrogen receptor-negative DCIS cases, 14/83 cases with dense TILs had local recurrence while only $1 / 33$ cases with sparse TILs experienced a recurrence.

When TILs were classified into three categories defined as: absent/very scanty TILs (mean number of touching TILs/DCIS duct $\leq 5$ cells), sparse TILs (from 6-20 cells/ DCIS duct) and dense TILs ( $>20$ cells/DCIS duct), recurrence occurred in $1 / 37$ patients (3\% of the group, $1.5 \%$ of the total recurrences), 23/258 patients (9\% of the group, $36.5 \%$ of the total recurrences), and $39 / 239$ (16\% of the group, $62 \%$ of the total recurrences), respectively.

In univariate survival analysis using two-tier classification system of TILs, dense TILs showed statistically significant association with shorter recurrence-free interval. This was observed when the analysis was conducted on the whole cohort $(p=0.002)$, or confined to patients treated with breast-conserving surgery $(p=0.001)$. In context of 
Table 3 Correlation between TILs density (based on mean number of touching TILs with cutoff 20 cells/DCIS duct) and clinicopathological parameters in the validation set

\begin{tabular}{llll}
\hline Parameter & \multicolumn{2}{l}{ TILs density } & Chi square $p$-value \\
\cline { 2 - 3 }$\left(\chi^{2}\right)$ & Dense $N$ & Sparse & \\
$(\%)$ & $N(\%)$ & \\
& & \\
\hline
\end{tabular}

Patient age

$\begin{array}{lllll}\leq 50 \text { years } & 71(52) & 65(48) & 4.09 & \mathbf{0 . 0 4 3} \\ >50 \text { years } & 168(42) & 230(58) & & \end{array}$

Presentation

Screening

$114(41) \quad 166(59)$

3.89

0.049

Symptomatic

125 (49)

$129(51)$

DCIS Size

$\begin{array}{lll}\leq 20 \mathrm{~mm} & 85(37) & 147(63) \\ >20 \mathrm{~mm} & 152(51) & 147(49)\end{array}$

10.66

0.001

DCIS Grade

\begin{tabular}{|c|c|c|c|c|}
\hline Low & $8(14)$ & $49(86)$ & 41.18 & $1.1 \times 10^{-9}$ \\
\hline Moderate & $44(33)$ & 88 (67) & & \\
\hline High & $187(54)$ & $158(46)$ & & \\
\hline
\end{tabular}

Comedo type necrosis

\begin{tabular}{|c|c|c|c|}
\hline Yes & $185(50)$ & $182(50) \quad 15.16$ & 0.00009 \\
\hline NO & $54(32)$ & $113(68)$ & \\
\hline
\end{tabular}

Associated Paget's disease

$\begin{array}{llll}\text { Yes } & 18(69) & 8(31) & 5.03 \\ \text { No } & 156(46) & 180(54)\end{array}$

0.025

Estrogen receptor status

$\begin{array}{lllll}\begin{array}{l}\text { Negative } \\ \text { Positive }\end{array} & 83(72) & 33(28) & 41.73 & \mathbf{1 . 0 4} \times \mathbf{1 0}^{-\mathbf{1 0}} \\ \begin{array}{l}\text { pe of DCIS } \\ \begin{array}{l}\text { Mixed with } \\ \text { vasion }\end{array}\end{array} & 105(80) & 27(20) & 51.29 & \mathbf{8 . 9 6} \times \mathbf{1 0}^{-\mathbf{1 3}} \\ \text { Pure DCIS } & 239(45) & 295(55) & & \end{array}$

Significant $p$ values are in bold

estrogen receptor status, dense TILs infiltrate was associated with shorter recurrence-free interval in both estrogen receptor-positive $(p=0.011)$ and estrogen receptornegative $(p=0.025)$ DCIS. Supporting the reproducibility of touching TILs evaluation in outcome prediction, univariate analysis using the second observer's scoring showed significant association with recurrence in the whole cohort and in patients treated with breast-conserving surgery ( $p=0.0001$ and $p=0.004$, respectively). Moreover, when the analysis was conducted using the average score between the two observers, it also showed significant association ( $p=0.002, p=0.01$ for the whole cohort and for patients treated with breast-conserving surgery, respectively).

Similar findings were observed when the three-tier classification system was used in the univariate analysis where dense TILs infiltrate was associated with shorter recurrence-
Table 4 Univariate association of TILs and other clinicopathological parameters with recurrence-free interval

\begin{tabular}{|c|c|c|}
\hline Parameter & Recurrence (\%) & $p$-value \\
\hline \multicolumn{3}{|l|}{ Patient age } \\
\hline$\leq 50$ years & $21(33)$ & 0.042 \\
\hline$>50$ years & $42(67)$ & \\
\hline \multicolumn{3}{|l|}{ Presentation } \\
\hline Symptomatic & $36(57)$ & 0.110 \\
\hline Screening & $27(43)$ & \\
\hline \multicolumn{3}{|l|}{ DCIS Size } \\
\hline$\leq 20 \mathrm{~mm}$ & $40(65)$ & 0.002 \\
\hline$>20 \mathrm{~mm}$ & $22(35)$ & \\
\hline \multicolumn{3}{|l|}{ DCIS Grade } \\
\hline Low & $3(5)$ & 0.409 \\
\hline Intermediate & $15(24)$ & \\
\hline High & $45(71)$ & \\
\hline \multicolumn{3}{|l|}{ Comedo type necrosis } \\
\hline No & $25(40)$ & 0.191 \\
\hline Yes & $38(60)$ & \\
\hline \multicolumn{3}{|l|}{ Associated Paget's disease } \\
\hline No & $32(89)$ & 0.552 \\
\hline Yes & $4(11)$ & \\
\hline \multicolumn{3}{|l|}{ Final operation type } \\
\hline Mastectomy & $20(32)$ & $1.1 \times 10^{-6}$ \\
\hline Breast-conserving surgery & $43(68)$ & \\
\hline \multicolumn{3}{|l|}{ Radiotherapy } \\
\hline No & $55(87)$ & 0.714 \\
\hline Yes & $8(13)$ & \\
\hline \multicolumn{3}{|l|}{ Estrogen receptor status } \\
\hline Negative & $15(27)$ & 0.992 \\
\hline Positive & $40(73)$ & \\
\hline \multicolumn{3}{|c|}{ TILs density (touching TILs) two-tier system ${ }^{a}$} \\
\hline Sparse & $24(38)$ & 0.002 \\
\hline Dense & $39(62)$ & \\
\hline \multicolumn{3}{|c|}{ TILs density (touching TILs) three-tier system ${ }^{a}$} \\
\hline Absent/very scanty & $1(1.5)$ & 0.005 \\
\hline Sparse & $23(36.5)$ & \\
\hline Dense & $39(62)$ & \\
\hline \multicolumn{3}{|l|}{ TILs density (stromal TILs) } \\
\hline Sparse & $47(75)$ & 0.117 \\
\hline Dense & $16(25)$ & \\
\hline
\end{tabular}

${ }^{\mathrm{a}}$ Classifications (definitions) of various touching TILs densities

Two-tier (two-groups) classified as Sparse where the mean number of TILs within the lesion is 20 cells/DCIS duct or less and Dense where the mean number of TILs within the lesion is more than 20 cells/DCIS duct

Three-tier (three-groups) classified as Absent/very scanty where the mean number of TILs within the lesion in $0-5$ cells/DCIS duct, Sparse where the mean number of TILs within the lesion is 6-20 cells/DCIS duct and Dense where the mean number of TILs within the lesion is more than 20 cells/DCIS duct

Significant $p$ values are in bold 

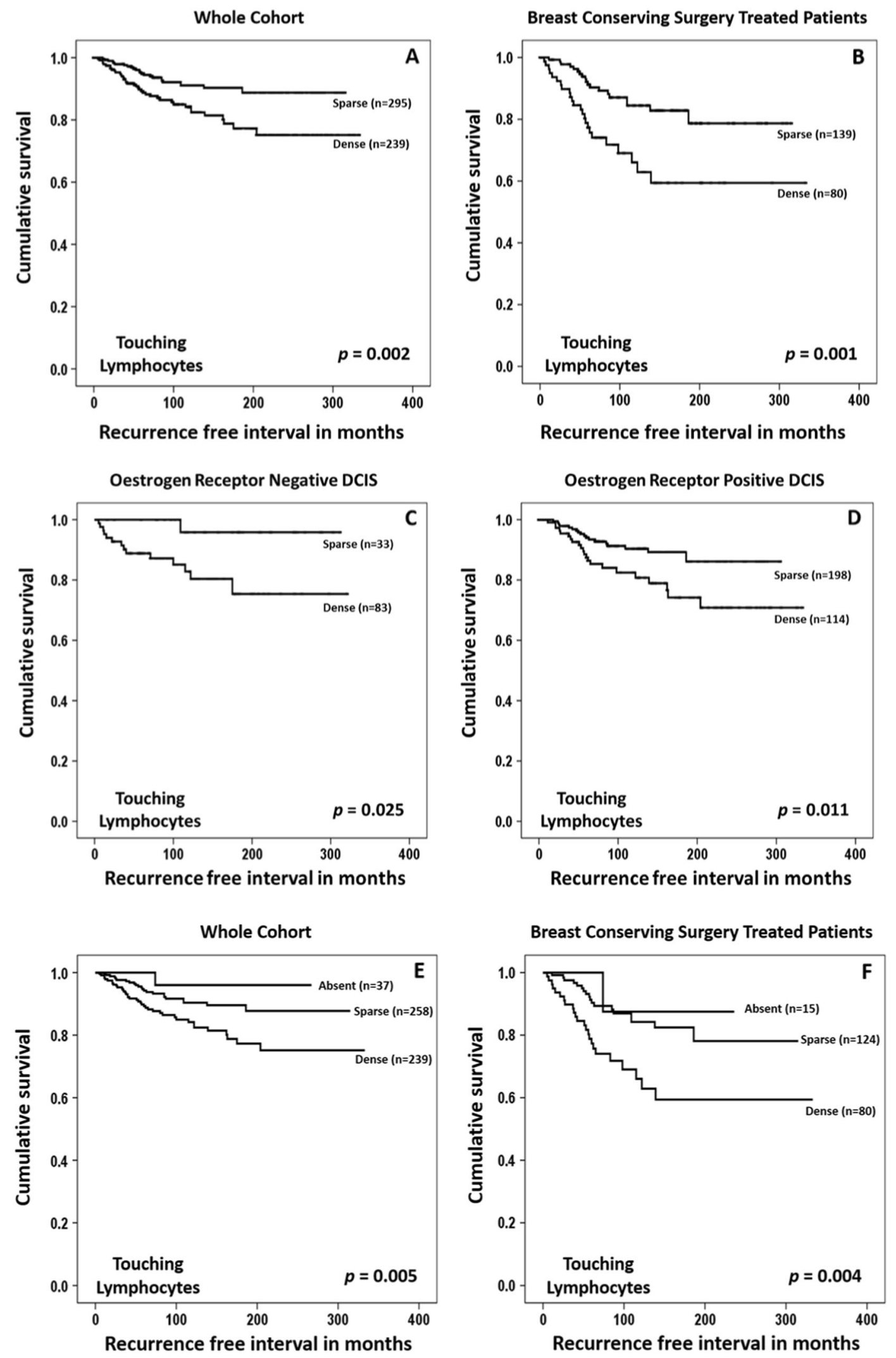

Fig. 3 Kaplan-Meier Curves showing association of touching TILs density (two-groups) with recurrence-free interval (in months). a All cases irrespective of surgical treatment, $\mathbf{b}$ cases treated with breast-

conserving surgery, $\mathbf{c}, \mathbf{d}$ according to estrogen receptor status, as well as when TILs density defined as three-groups in e the whole cohort, and $\mathbf{f}$ breast-conserving surgery-treated patients 
Table 5 Multivariate analysis results (Cox regression model)

\begin{tabular}{lllll}
\hline Parameters & $\begin{array}{l}\text { Hazard ratio } \\
\text { (HR) }\end{array}$ & $\begin{array}{l}\text { 95.0\% } \\
\text { confident } \\
\text { interval }(\mathrm{CI})\end{array}$ & $\begin{array}{l}\text { Significance } \\
p \text {-value }\end{array}$ \\
\cline { 2 - 4 } & & \multicolumn{2}{l}{$\begin{array}{l}\text { Lower } \\
\text { Upper }\end{array}$} \\
\hline Patient age & 0.599 & 0.318 & 1.127 & 0.112 \\
DCIS size & 0.755 & 0.403 & 1.415 & 0.381 \\
$\begin{array}{l}\text { DCIS grade } \\
\text { Comedo type }\end{array}$ & 1.531 & 0.899 & 2.608 & 0.117 \\
necrosis & 0.639 & 0.341 & 1.197 & 0.162 \\
$\begin{array}{l}\text { Radiotherapy } \\
\text { Estrogen receptor }\end{array}$ & 0.423 & 0.172 & 1.037 & 0.060 \\
status & 0.934 & 0.477 & 1.829 & 0.841 \\
Dense TILs & 2.573 & 1.412 & 4.690 & $\mathbf{0 . 0 0 2}$
\end{tabular}

Multivariate analysis results (Cox regression model) performed for patients treated with Breast conservative surgery only

Significant $p$ values are in bold

free interval in the whole cohort and breast-conserving surgery-treated patients $(p=0.005$ and $p=0.004$; respectively). The detailed results of univariate analyses between different clinicopathological parameters as well as TILs against recurrence-free interval, and Kaplan-Meier curves are provided in Table 4 and Fig. 3, respectively. Interestingly, there was no statistically significant association between recurrence-free interval and TILs density when assessed based on the percentage of overall stromal TILs ( $p=0.117$ for the whole cohort and $p=0.138$ for patients treated with breast-conserving surgery).

Cox regression multivariate model with other clinicopathological parameters including patient's age, tumor size, nuclear grade, presence of comedo necrosis, adjuvant radiotherapy, and estrogen receptor status showed that dense touching TILs is the only independent predictor of shorter recurrence-free interval in patients treated with breast-conserving surgery (hazard ratio $=2.6,95 \%$ confident interval $=1.41-4.7, p=0.002)$ (Table 5).

\section{Discussion}

Currently, clinicopathological parameters used for DCIS risk-stratification such as age, nuclear grade, tumor size, and associated comedo necrosis are still insufficient to accurately estimate recurrence risk associated with DCIS $[21,22]$. TILs in DCIS are thought to have a role in tumor behavior and progression; however, there are currently no consensus guidelines to evaluate TILs in clinical practice. Previous studies of TILs evaluation in DCIS neither used clear or uniform definition of the stromal area surrounding DCIS for TILs assessment nor identified cutoff points that can prognostically stratify DCIS. Some studies used the
International Working TILs Group guidelines [14] with modification to DCIS and assessed percentage of stromal TILs at the stroma within the boundaries of the whole lesion $[5,19]$. Pruneri et al. [15] defined stromal TILs surrounding DCIS as those located at the area within two high-power microscopic fields form the DCIS ducts and this method was adopted by other authors [17, 18, 23]. These studies have reported lack of association between TILs density and DCIS recurrence. In the current study, TILs were assessed in a large cohort of DCIS with long-term follow-up data using various scoring methods to determine the most reproducible method that can additionally provide prognostic value. Prognostic stratification system has also been proposed to facilitate TILs application in DCIS in routine practice.

Assessment of TILs in the training set revealed that touching TILs is the optimal method for TILs scoring in DCIS. This conclusion was based on several criteria. Firstly, touching TILs had the highest concordance rate between the observers with or without prior methodology discussion. Secondly, they were positively correlated with TILs in the other topographic areas, hence representing TILs density within the whole lesion without requirement to assess TILs in wider areas around ducts, which is time consuming. Thirdly, touching TILs assessment was the easiest and fastest method, which can be performed without the need for accurate measurement of distance around ducts or adjustment of the scale and area's dimensions. Moreover, assessment of touching TILs avoids confusion/variability in scoring cells within overlapping areas between adjacent ducts. Lastly, touching TILs showed the strongest significant association with other prognostic clinicopathological parameters and DCIS outcome (recurrencefree interval).

In this study, touching TILs showed significant association with recurrence-free interval in both training and validation cohorts, as well as when the analysis was conducted using different observers' scores. Notably, dense touching TILs were associated with shorter recurrence-free interval when the analysis was performed either in the whole cohort or when confined only to patients treated with breast-conserving surgery. Importantly, touching TILs was an independent prognostic factor for DCIS recurrence in patients treated with breast-conserving surgery regardless of other known determinants of tumor behavior [24-27].

Interestingly, the percentage of overall stromal TILs assessed using the same methods for TILs evaluation described in the previous studies [5, 15, 17-19] showed not only the least concordance rates but also variability in association with the outcome. Unlike stromal TILs evaluation in invasive breast carcinoma, the low inter-observer and intra-observers' agreement may reflect the subjectivity 
of assessment of stromal TILs in DCIS, even with the use of predefined criteria of stromal area surrounding DCIS.

Studies of TILs in invasive breast carcinoma reported associations between TILs and better prognosis especially in triple-negative breast cancer and supported their synergistic effect with chemotherapy [12, 13]. By contrast, the current study demonstrates that dense TILs in DCIS are associated not only with other potential risk factors for aggressive DCIS behavior but also with increased risk of tumor recurrence and progression. Supporting our results, Pruneri et al. reported that dense TILs are correlated with more aggressive DCIS [15]. Hendry et al. [17] have also reported dense TILs are associated with high grade, comedo type, estrogen receptor-negative, and Her2-positive DCIS lesions. Although both studies failed to find a significant association between TILs density and tumor outcome, this might be due to different assessment methodology. A recent molecular study showed that dense TILs were associated with aggressive DCIS. Copy number variation in DCIS with dense TILs was shown to be more profound than lesions with low TILs density, which might indicate the higher immunoediting capability of DCIS with dense TILs making them more likely to progress and recur [17]. In addition, dense TILs were associated with high DCIS Oncotype DX score [28], providing evidence to support our results that dense TILs are associated with poor outcomes in DCIS. Association between dense TILs infiltrate and poor prognosis has also been reported in oral, colonic, prostatic, and pancreatic preinvasive neoplasia [29, 30].

Interestingly, dense TILs were associated with shorter recurrence-free interval irrespective of estrogen receptor expression in DCIS. This might indicate that the crosstalk between the immune microenvironment and tumor cells contributing to DCIS recurrence and/or progression is unrelated to estrogen receptor pathway. Comparing TILs density in DCIS associated with invasion with pure DCIS indicates more TILs density in the former. This finding supports the hypothesized role of inflammatory cells in DCIS progression and aggressiveness [5, 16, 31, 32]. TILs density assessment and reporting in the routine practice for DCIS diagnosed by core biopsy may provide a predictive factor for presence of invasion in these lesions as previously observed [33, 34].

Involvement of different immune cell subpopulations in DCIS behavior has been speculated. Regulatory T cells (Tregs) play key roles in tumor evasion from the immune system [35]. Homeostasis of the immune response in the body is regulated by T-regs, however, a paradoxical action may occur through over-suppression of the immune cells attacking the tumor cells leading to tumor progression [36]. It was shown that high-grade DCIS lesions harbor higher percentage of FOXP3 + cells [19]. Moreover, tumor cells surrounded by dense TILs may produce some protective proteins to evade the host immune system. Thompson et al. [5] have reported that DCIS with dense TILs show higher level of programmed cell death ligand 1 (PDL-1)-positive tumor cells. This was supported with two similar studies that characterized immune microenvironment in DCIS [17, 19]. Although the role of B-lymphocytes in tumor immunity and behavior is unclear, a study on a small cohort of DCIS by Miligy et al. [37], showed increased B-lymphocytes infiltrate was associated with increased recurrence liability and with other poor outcome parameters.

In conclusion, using touching TILs as an assessment method for TILs in hematoxylin and eosin-stained full-face DCIS sections is a reproducible and practical method to predict tumor behavior and progression. Application of this method in routine practice would aid in risk-stratification of DCIS for improved, individualized management.

Acknowledgements We thank the Nottingham Health Science Biobank and Breast Cancer Now Tissue Bank for provision of tissue samples.

\section{Compliance with ethical standards}

Conflict of interest The authors declare that they have no conflict of interest.

\section{References}

1. Lee RJ, Vallow LA, McLaughlin SA, et al. Ductal carcinoma in situ of the breast. Int J Surg Oncol. 2012;2012:123549. https:// doi.org/10.1155/2012/123549.

2. Siegel RL, Miller KD, Jemal A. Cancer statistics, 2016. CA Cancer J Clin. 2016;66:7-30.

3. Francis A, Thomas J, Fallowfield L, et al. Addressing overtreatment of screen detected DCIS; the LORIS trial. Eur J Cancer. 2015;51:2296-303.

4. Carraro DM, Elias EV, Andrade VP. Ductal carcinoma in situ of the breast: morphological and molecular features implicated in progression. Biosci Rep. 2014;34:e00090.

5. Thompson E, Taube JM, Elwood H, et al. The immune microenvironment of breast ductal carcinoma in situ. Mod Pathol. 2016;29:249-58.

6. Muggerud AA, Hallett M, Johnsen $\mathrm{H}$, et al. Molecular diversity in ductal carcinoma in situ (DCIS) and early invasive breast cancer. Mol Oncol. 2010;4:357-68.

7. Van Bockstal M, Lambein K, Gevaert O, et al. Stromal architecture and periductal decorin are potential prognostic markers for ipsilateral locoregional recurrence in ductal carcinoma in situ of the breast. Histopathology. 2013;63:520-33.

8. Rudloff U, Jacks LM, Goldberg JI, et al. Nomogram for predicting the risk of local recurrence after breast-conserving surgery for ductal carcinoma in situ. J Clin Oncol. 2010;28:3762-9.

9. Solin LJ, Gray R, Baehner FL, et al. A multigene expression assay to predict local recurrence risk for ductal carcinoma in situ of the breast. J Natl Cancer Inst. 2013;105:701-10.

10. Sabatier R, Finetti P, Mamessier E, et al. Prognostic and predictive value of PDL1 expression in breast cancer. Oncotarget. 2015;6:5449-64. 
11. Pardoll DM. The blockade of immune checkpoints in cancer immunotherapy. Nat Rev Cancer. 2012;12:252-64.

12. Adams S, Gray RJ, Demaria S, et al. Prognostic value of tumor-infiltrating lymphocytes in triple-negative breast cancers from two Phase III Randomized Adjuvant Breast Cancer Trials: ECOG 2197 and ECOG 1199. J Clin Oncol. 2014;32:2959-66.

13. Ibrahim EM, Al-Foheidi ME, Al-Mansour MM, Kazkaz GA. The prognostic value of tumor-infiltrating lymphocytes in triplenegative breast cancer: a meta-analysis. Breast Cancer Res Treat. 2014;148:467-76.

14. Salgado R, Denkert C, Demaria S, et al. The evaluation of tumorinfiltrating lymphocytes (TILs) in breast cancer: recommendations by an International TILs Working Group 2014. Ann Oncol. 2015;26:259-71.

15. Pruneri G, Lazzeroni M, Bagnardi V, et al. The prevalence and clinical relevance of tumor-infiltrating lymphocytes (TILs) in ductal carcinoma in situ of the breast. Ann Oncol. 2017;28:321-8

16. Man YG, Stojadinovic A, Mason J, et al. Tumor-infiltrating immune cells promoting tumor invasion and metastasis: existing theories. J Cancer. 2013;4:84-95.

17. Hendry S, Pang JB, Byrne DJ, et al. Relationship of the breast ductal carcinoma in situ immune microenvironment with clinicopathological and genetic features. Clin Cancer Res. 2017;23:5210-7.

18. Hendry $S$, Salgado R, Gevaert $T$, et al. Assessing tumorinfiltrating lymphocytes in solid tumors: A practical review for pathologists and proposal for a standardized method from the International Immuno-Oncology Biomarkers Working Group: Part 2: TILs in melanoma, gastrointestinal tract carcinomas, nonsmall cell lung carcinoma and mesothelioma, endometrial and ovarian carcinomas, squamous cell carcinoma of the head and neck, genitourinary carcinomas, and primary brain tumors. Adv Anat Pathol. 2017;24:311-35.

19. Campbell MJ, Baehner F, O'Meara T, et al. Characterizing the immune microenvironment in high-risk ductal carcinoma in situ of the breast. Breast Cancer Res Treat. 2017;161:17-28.

20. Lee N, Zakka LR, Mihm MC Jr., Schatton T. Tumour-infiltrating lymphocytes in melanoma prognosis and cancer immunotherapy. Pathology. 2016;48:177-87.

21. Furnival C. Ductal carcinoma in situ of the breast: present and future. ANZ J Surg. 2006;76:4-5.

22. Virnig BA, Tuttle TM, Shamliyan T, Kane RL. Ductal carcinoma in situ of the breast: a systematic review of incidence, treatment, and outcomes. J Natl Cancer Inst. 2010;102:170-8.

23. Dieci MV, Radosevic-Robin N, Fineberg S, et al. Update on tumor-infiltrating lymphocytes (TILs) in breast cancer, including recommendations to assess TILs in residual disease after neoadjuvant therapy and in carcinoma in situ: A report of the International Immuno-Oncology Biomarker Working Group on Breast Cancer. Semin Cancer Biol. 2017. https://doi.org/10.1016/j.semca ncer.2017.10.003
24. Kong I, Narod SA, Taylor C, et al. Age at diagnosis predicts local recurrence in women treated with breast-conserving surgery and postoperative radiation therapy for ductal carcinoma in situ: a population-based outcomes analysis. Curr Oncol. 2014;21: e96-104.

25. Kerlikowske K, Molinaro A, Cha I, et al. Characteristics associated with recurrence among women with ductal carcinoma in situ treated by lumpectomy. J Natl Cancer Inst. 2003;95:1692-702.

26. Stallard S, Hole DA, Purushotham AD, et al. Ductal carcinoma in situ of the breast -- among factors predicting for recurrence, distance from the nipple is important. Eur J Surg Oncol. 2001;27:373-7.

27. Mechera R, Viehl CT, Oertli D. Factors predicting in-breast tumor recurrence after breast-conserving surgery. Breast Cancer Res Treat. 2009;116:171-7.

28. Knopfelmacher A, Fox J, Lo Y, Shapiro N, Fineberg S. Correlation of histopathologic features of ductal carcinoma in situ of the breast with the oncotype DX DCIS score. Mod Pathol. 2015;28:1167-73.

29. Gannot G, Gannot I, Vered H, Buchner A, Keisari Y. Increase in immune cell infiltration with progression of oral epithelium from hyperkeratosis to dysplasia and carcinoma. $\mathrm{Br} \mathrm{J}$ Cancer. 2002;86:1444-8

30. Hiraoka N, Onozato K, Kosuge T, Hirohashi S. Prevalence of FOXP3 + regulatory $\mathrm{T}$ cells increases during the progression of pancreatic ductal adenocarcinoma and its premalignant lesions. Clin Cancer Res. 2006;12:5423-34.

31. Man YG, Tai L, Barner R, et al. Cell clusters overlying focally disrupted mammary myoepithelial cell layers and adjacent cells within the same duct display different immunohistochemical and genetic features: implications for tumor progression and invasion. Breast Cancer Res. 2003;5:R231-41.

32. Jiang B, Mason J, Jewett A, et al. Tumor-infiltrating immune cells: triggers for tumor capsule disruption and tumor progression? Int J Med Sci. 2013;10:475-97.

33. Lee AH, Gillett CE, Ryder K, et al. Different patterns of inflammation and prognosis in invasive carcinoma of the breast. Histopathology. 2006;48:692-701.

34. Doebar SC, de Monye C, Stoop H, et al. Ductal carcinoma in situ diagnosed by breast needle biopsy: Predictors of invasion in the excision specimen. Breast. 2016;27:15-21.

35. Gobert M, Treilleux I, Bendriss-Vermare N, et al. Regulatory $\mathrm{T}$ cells recruited through CCL22/CCR4 are selectively activated in lymphoid infiltrates surrounding primary breast tumors and lead to an adverse clinical outcome. Cancer Res. 2009;69:2000-9.

36. Curiel TJ, Coukos G, Zou L, et al. Specific recruitment of regulatory $\mathrm{T}$ cells in ovarian carcinoma fosters immune privilege and predicts reduced survival. Nat Med. 2004;10:942-9.

37. Miligy I, Mohan P, Gaber A, et al. Prognostic significance of tumour infiltrating B lymphocytes in breast ductal carcinoma in situ. Histopathology. 2017;71:258-68. 